\title{
Design of a new power management unit used for high voltage watt-hour meters
}

\author{
CHENG Yingying, DU Jie, XIAO Ji, HOU Xingzhe, ZHOU Feng
}

State Grid Chongqing Electric Power CO. Electric Power Research Institute

\begin{abstract}
Keywords: Power transformer, online winding diagnosis, frequency response analysis, repetitive impulse
\end{abstract}

Abstract: New high voltage watt-hour meters have the advantages of high precision measurement, low power consumption, information intelligence, etc. which are selected in more and more applications. This paper researched on the power supply of the new metering devices, designed a new power management unit based on induction current energy harvesting. The unit introduced a smart electrical energy storage function between the voltage regulator and the energy harvesting coil, made sure the metering devices can operate stably, avoided the metering losses due to the power off of the metering devices. The experimental results showed that the storage electrical energy could supply for 4.5 hours without the energy harvesting.

\section{Introduction}

As the measure device employed by power producer, power seller and power consumer, the plays a significant role in power system and has been widely used. Therefore, the safety, reliability and accuracy of the watt-hour meter needs to be guaranteed strongly. Traditional watt-hour meter that consists of current transformer, voltage transformer and electricity meter has some disadvantages of high power consumption, large error, material waste, electricity easily stolen and so on. With the great development of many techniques such as measuring sensor, communication, information technology, an advanced power meter has been developed. By setting the measuring circuit hanging on the HV side, this new type of power meter can prevent the electricity stolen. In this paper, to distinguish with the traditional watt-hour meter, this new type of watt-hour meter is called high voltage watt-hour meter. The high voltage watt-hour meter mainly bases on the micro controller to realize the measurement, data monitoring and communication. It has advantages of high intelligence and accuracy. To guarantee the stable operation of the high voltage meter, it is necessary for the micro controller and other integrated chip to obtain supply power at the status of high voltage. Currently, the power supply mode mainly contains method below.

The first method is solar power supply. The method is mature, clean pollution-free, renewable, in line with the requirements of smart grid. However, solar panels are heavy equipment which are vulnerable to light, ambient temperature, day and night, dust and other factors. The power generation uncertainty limits its large-scale applications. The second method is laser power, which generates light energy at high-power lasers located at ground potentials, and then transmits them to the high-voltage region through energy-transmitting optical fibers and then converts them into electrical energy through optical power conversion modules. The advantages of this method are stable power supply, hardly influenced by electromagnetic interference and power network operating state; the disadvantage is the low power conversion efficiency, low high power laser lifetime, and that the equipment is expensive. The third method is the usage of capacitive divider to take energy, which is a currently widely used secure power supply program. In the energy requirements of high-voltage energy meter, the "phase voltage" can be employed to obtain the high-voltage equipotential working power. The method has a wide range of voltage to adapt to the rated voltage of $50 \%$ to $200 \%$, over which it can maintain the stability of work and meanwhile the equipment is light and efficient. The final method is the usage of the induction current to take energy. This technology is a more widely used technology with many advantages. With the effective use of high-voltage wire around the alternating magnetic field, it can take electricity based on the electromagnetic induction principle, and 
then rectified and filtered; steady electricity can be supplied to function modules of the metering device. The method has advantages of rational use of available resources, energy saving, small size, low cost, the safe and convenient usage.

In power supply requirements of the high-voltage energy meter, there are still some technical problems on the use of induced current to take energy technology: (1) large current energy leakage protection, how to design protection circuit and transformer is a large difficulty; (2) when the bus current is close to no-load condition, how to ensure the stable supply of power, how to ensure the immediate measurement when the power supply is recovered. In this paper, the induction current method are used for high-voltage energy meter power supply. Bases on the analysis of the existing energy meter power consumption, referring to the above two issues, a new power management unit is designed to make the technology more practical.

\section{Analysis on High-voltage Energy Meter Power Consumption}

Energy meter is a power metering device, but it is also a power consumer. At present, China's online use of various types of energy meter is about hundreds of millions, their power consumption vary in a widely range. According to the reference [5], the power consumption of each type of electric energy meter is introduced as follows:

1) Induction meter, the market share is about $15 \%$ to $20 \%$, its own power consumption in about $1.7 \mathrm{~W}$;

2) Electronic energy meter, the market share is about $50 \%$ or more, its own power consumption is about $1 \mathrm{~W}$ to $1.5 \mathrm{~W}$;

3) Smart energy meter, the market share is about $20 \%$ to $30 \%$, and dissipation of its power consumption is very large, the minimum type is $0.2 \mathrm{~W}$, the maximum type is $1.4 \mathrm{~W}$, most of them are around $1.1 \mathrm{~W}$.

The energy meter introduced in this paper uses the micro-controller to achieve energy metering, its power consumption is approximately equal to the smart energy meter. According to the State Grid Standard Q/GDW-364-2009 and Q/GDW-362-2009, the power consumption of smart meters should be no more than $1.5 \mathrm{~W}$. Combining with the data of reference [5], we can see that although the maximum power consumption stipulated in the national network standard has its rationality, there are still problems of over low standard. In order to improve product competitiveness, the manufacturers will certainly try to reduce the power consumption of the energy meter. According to the above analysis, the power management unit designed in this paper is assumed to be $1 \mathrm{~W}$ to meet the normal power supply demand of most high-quality energy meters. In addition, the paper also defaults that when the bus power failure or power outage is repaired, the meter will automatically enter the low power consumption status through intelligent judgment and the output power is set to be $0.5 \mathrm{~W}$.

\section{Power Management Unit}

\section{System Structure and Schematic Diagram}

The section headings are in boldface capital and lowercase letters. Second level headings are typed as part of the succeeding paragraph (like the subsection heading of this paragraph). As the bus current has factors of large current fluctuation range, instability and so on, the induction voltage of the coil and output power is both very unstable. How to design and optimize the most simple, reliable power management circuit, to realize the effective management of the unstable input voltage source, and then output stable and reliable DC power supply voltage, has been the goal of many technical personnel in recent years.

Figure 1 shows the schematic diagram of high-voltage energy meter's management unit designed for the power supply demand, based on the induction current to take power, in which the 
energy-taking device is the energy source of power management unit; The rectifier module converts the induced alternating current into the direct current which is needed by the subsequent circuit and is stored in the storage capacitor. The voltage hysteresis comparator module controls the on-off of the voltage regulator input terminal intelligently by designing relevant parameters to ensure that the subsequent circuit can work stably. Regulator is responsible for the power supply circuit, directly providing a standard stable DC voltage to the high-voltage energy meter module.

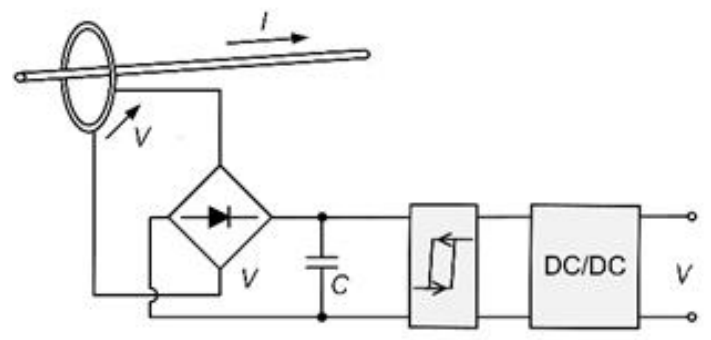

Fig.1 Structure and schematic diagram of power management unit

When the bus current is $10 \mathrm{~A}$, the maximum output power of the induction coil should be greater than $1 \mathrm{~W}$. The $\mathrm{AC}$ output $\mathrm{V}_{\text {induction }}$ of the coil is firstly converted into direct current by a full bridge rectifying circuit and stored in the storage capacitor $\mathrm{C}_{\text {storage }}$, and an intelligent energy storage module is added between the $\mathrm{C}_{\text {storage }}$ and the input terminal of the voltage regulator, to control the connection between the storage capacitor and the input voltage regulator, ensuring stable operation of the regulator chip to guarantee that when the transmission bus is recovered to power on, the energy meter can immediately start to work.

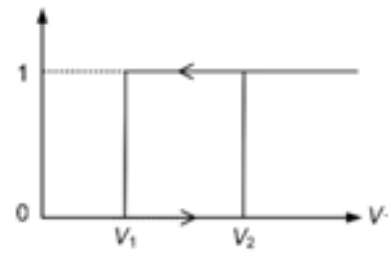

Fig.2 Schematic diagram of energy storage capacitor output

Fig. 2 shows the connection between the storage capacitor and the voltage regulator, the function is achieved by the hysteresis voltage comparator. In the initial stage, the voltage at both ends of $\mathrm{C}_{\text {storage }}$ must reach the voltage $V_{2}$ to provide output. If the output power of the energy-saving coil is greater than the power consumption of the energy meter, the energy storage voltage $V_{\text {storage }}$ will continue to rise, but not exceed the designed protection voltage. If the coil's output power is zero or less than the power consumption, the storage voltage $\mathrm{V}_{\text {storage }}$ will fall, but the regulator can continue to work until the voltage drops to $\mathrm{V}_{1}$. So the storage capacitor should be able to store at least the electric quantity $\mathrm{E}_{\min }$, as shown in equation (1). By this formula, it can be seen that in order to increase the storage capacity, it can be achieved by increasing the capacity of $\mathrm{C}_{\text {storage }}$ and voltage $\mathrm{V}_{2}$.

$$
E_{\text {min }}=\frac{1}{2} C_{\text {储能 }}\left(V_{2}^{2}-V_{1}^{2}\right)
$$

\section{Implementation of Management Unit Circuit}

As the output of the coil is AC, so at first it should go through the bridge finishing circuit to be converted to DC current and then stored in the energy storage capacitor shown in Fig.3. Two-way VR tube at input terminal of voltage regulator and $36 \mathrm{~V}$ unidirectional $\mathrm{VR}$ tube at output terminal is mainly used for high-current energy protection and follow-up chip protection. Storage capacitor selects ten $2.7 \mathrm{~V}, 500 \mathrm{~F}$ super-farad capacitor in series, which can enhance the storage voltage. Compared to the parallel capacitor group, its theoretical storage energy increased by 10 times. The volume of the super-farad capacitor is similar to the No. 1 cell $(35 \times 60 \mathrm{~mm})$, which is acceptable in the design of the high-voltage energy meter. 


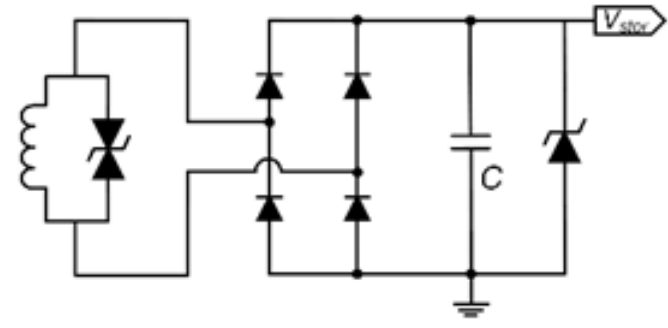

Fig.3 Rectifier circuit

For the DC / DC power regulator chip to work properly, and when the power lines is recovered to power on, the energy meter can immediately start the measurement, the voltage hysteresis comparator between the regulator and the storage capacitor is necessary. Figure 4 shows schematic diagram of the functional circuit. The reference voltage VR $(+5 \mathrm{~V})$ is provided by the chip LTC3330. VR also serves as power supply voltage for the comparator chip LT1716. Regarding that the power consumption of chip LT1716 is very low, the input source of the reference voltage is provided by small coil, LTC3330 chip manual provides a more complete reference circuit on this part of the design[7].

In the hysteresis comparator, the resistance of the resistance $\mathrm{R}_{2}$ is $330 \mathrm{~K}$, of $\mathrm{R} 3$ is $1 \mathrm{M}$. The two resistance values are large, which is conducive to reducing the power consumption of the storage capacitor in the branch. Meanwhile, the resistance of $\mathrm{R}_{3}$ is 3.03 times of $\mathrm{R}_{2}$, so in the hysteresis comparator, $\mathrm{V}_{1}$ is $5 \mathrm{~V}, \mathrm{~V}_{2}$ is $20.15 \mathrm{~V}$. According to equation (1), it can be calculated that when the power management circuit starts to work, the storage capacitor stores at least $9525.6 \mathrm{~J}$ of power for subsequent circuit consumption, regardless of the regulator conversion efficiency, assuming that the power meter is $0.5 \mathrm{~W}$, the theoretical operation time length of voltage regulator is 5.3 hours.

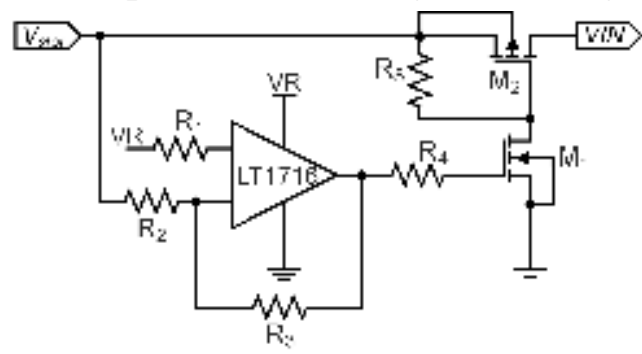

Fig.4 Hysteresis comparator circuit

The regulator chip in power management unit selects LT3971, The chip can provide +5 V DC stabilized voltage and output current up to $1.2 \mathrm{~A}$, which can fully meet the power meter power supply needs. Fig.5 shows the voltage regulator circuit. Its design is simple, and the output voltage is stable.

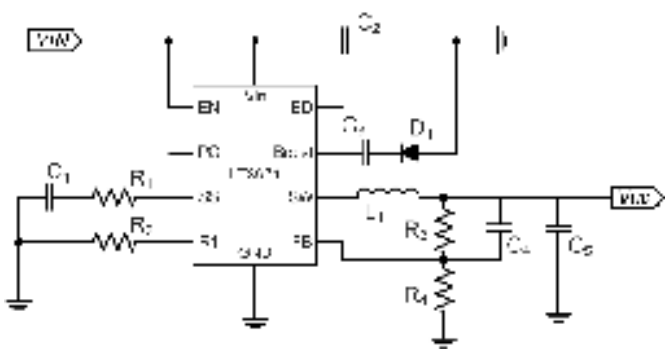

Fig.5 Voltage regulator circuit

\section{Functional Verification of Power Management Unit}

To verify the above design, the experiment uses $220 \mathrm{~V}$ AC power supply connected with rheostat $(10 \sim 100 \Omega)$ to analog transmission line current. Considering that the output of the regulator is DC voltage, so the pure resistance of $50 \Omega$ is chosen to analog power consumption of the energy meter. 


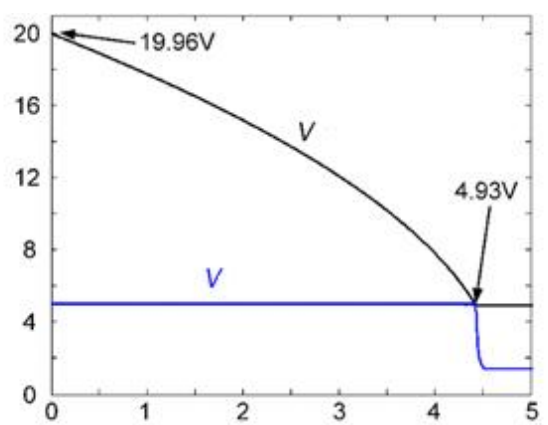

Fig.6 Waveform of power management unit storage voltage and supply voltage

Figure 6 shows the power management unit input and output voltage waveform. First, a current transmission line is placed inside the taking coil, as the hysteresis comparator is designed to control the on-off of the input, the regulator does not work at the moment, and the energy is stored completely in the super capacitor. The energy storage voltage rises from 0 to $20.15 \mathrm{~V}$. Meanwhile, the hysteresis comparator outputs high voltage, controlling input switch M2 (shown in Figure 4) to close, and the

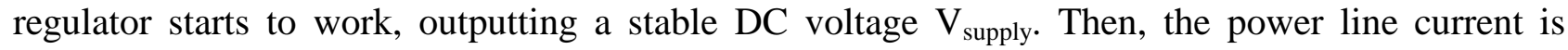
disconnected, measuring the actual work time length of the power management unit.

Use the real-time control system dSPACE (DS1103) and Matlab software to measure the storage and supply voltage. The sampling rate is $100 \mathrm{~Hz}$. But in practical, to compress the data, the software automatically calculate the average of 100 points and then store the data, so the actual sampling rate is $1 \mathrm{~Hz}$, that is, a voltage value per second. The final voltage waveform curve is shown in Fig.6. From it, it can be seen that when the energy storage voltage $\mathrm{V}_{\text {storage }}$ decreases to $4.93 \mathrm{~V}$, the output of the voltage hysteresis comparator jumps to the low level, the switch M2 at the input end opens, The regulator is no longer operating. Through the experimental results, it can be seen that when the power line is in outage, the power management unit can work stably for at least 4.5 hours, which is shorter than the theoretical calculation of 5.3 hours. This is mainly due to the theoretical calculation does not take into account the conversion efficiency of the regulator and the power consumption of the voltage hysteresis comparator's input end.

\section{Conclusion}

This paper firstly analyzes the power demand of the new high-voltage electric energy meter. Then, a set of power management unit based on induced current for power supply is developed, which includes functions of rectification, current limit protection, undercurrent protection, DC / DC regulation and so on. Finally, the stability of the power management unit is tested in a laboratory environment. Finally, the stability of the power management unit is tested in a laboratory environment. The results show that if no current passes through the transmission bus, the stored energy can guarantee stable operation for 4.5 hours, ensuring that when the bus is recovered to power on, the metering device can immediately start energy measurement without loss of stored data. In addition, the power management unit does not use ordinary battery storage technology, in line with today's green development values. The technology has very high application prospects.

\section{References}

[1] Peng Ling. Asset management of energy metering device [J] .Chinese high-tech enterprises, 2015, 01: $185-187$.

[2] WEN He, TENG Zhaosheng. Improved electric power measurement method and its application while harm oink exists[J].Chinese journal of scientific insutrument.2011,32(1).

[3] LAN Jibin, XU Yang. Fuzzy analytic hierarchy weights study[J].System engineering theory \& practice, 2006.

[4] MA Weiqing, YU Yaozhang. The status evaluation of power grid equipment based on Dynamic Weight Analytic Hierarchy Process dynamic weight analytic hierarchy process[J].Electronic test,2015. 